\title{
The Polarized Gamma-Ray Observer, PoGOLite
}

\author{
By Hiromitsu TAKAhashi ${ }^{1)}$, Masayuki MATSUOKA ${ }^{1)}$, Yudai UMEKI ${ }^{1)}$, Hiroaki YoshidA ${ }^{1)}$, Takuya TANAKA ${ }^{1)}$, Tsunefumi \\ MizUnO $^{1)}$, Yasushi FuKAZAWA ${ }^{1)}$, Tuneyoshi KAMAE ${ }^{2)}$, Grzegorz MADEJSKI ${ }^{2)}$, Hiroyasu TAJimA ${ }^{2)}$, Mózsi Kiss ${ }^{3)}$, \\ Wlodzimierz KLAMrA ${ }^{3)}$, Stefan LARSSON ${ }^{3)}$, Cecilia MARINI BetTolo ${ }^{3)}$, Mark PEARCE ${ }^{3)}$, Felix RYdE ${ }^{3)}$, Stefan RYDSTRÖM $^{3)}$, \\ Kohei Kurita $^{4)}$, Yoshikazu Kanai ${ }^{4)}$, Makoto Arimoto ${ }^{4)}$, Masaru Ueno $^{4)}$, Jun KataokA ${ }^{4)}$, Nobuyuki Kawai ${ }^{4)}$, Magnus

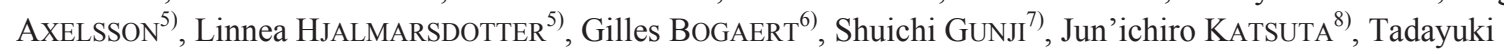 \\ TAKAHASHI $^{8)}$, Gary VARNER ${ }^{9)}$ and Takayuki YUASA ${ }^{10)}$

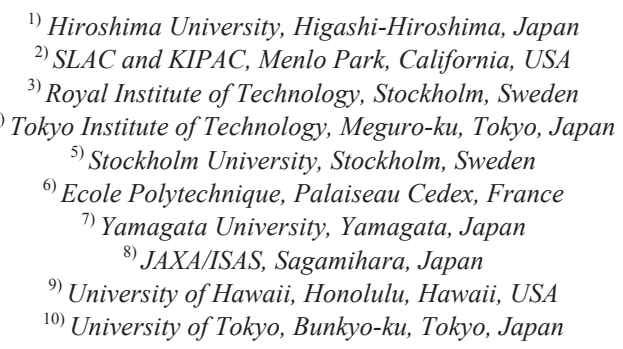

(Received July 17th, 2009)

The Polarized Gamma-ray Observer, PoGOLite, is a balloon experiment with the capability of detecting $10 \%$ polarization from a $200 \mathrm{mCrab}$ celestial object in the energy-range $25-80 \mathrm{keV}$. During a beam test at KEK-PF in 2008,19 detector units and one anti-coincidence detector were assembled, and a $50 \mathrm{keV}$ X-ray beam with a polarization degree of $\sim 90 \%$ was irradiated at the center unit. Signals from all 20 units were fed into flight-version electronics consisting of six circuit boards (four waveform digitizer boards, one digital I/O board and one router board) and one microprocessor (SpaceCube), which communicate using a SpaceWire interface. One digitizer board, which can associate up to 8 detectors, outputs a trigger signal. The digital $\mathrm{I} / \mathrm{O}$ board handles the trigger and returns a data acquisition request if there is no veto signal (upper or pulse-shape discriminators) from any detector unit. This data acquisition system worked well, and the modulation factor was successfully measured to be $\sim 34 \%$. These results confirmed the capabilities of the data-acquisition system for a "pathfinder" flight planned in 2010.

Key Words: Balloon Experiment, Polarization Measurement, Hard X-Ray, Soft Gamma-Ray, SpaceWire Interface

\section{Introduction}

Polarization measurements of celestial objects in hard $\mathrm{X}$-rays/soft gamma-rays have been expected to provide a powerful probe into high-energy emission mechanism as well as the distribution of magnetic field and matter around the sources such as pulsars, black hole binaries, highly magnetized neutron stars, micro-quasars and active galactic nuclei. Despite the importance of these measurements, polarization from only the Crab nebula has been measured at $2.6 \mathrm{keV}$ and $5.2 \mathrm{keV}^{1)}$, and recently above $200 \mathrm{keV}^{2)}$. Additional measurements in several $\mathrm{keV}$ range, both of this source and of other objects, are crucial for the detailed understanding of the emission mechanisms in these objects.

The Polarized Gamma-ray Observer (PoGOLite) is a balloon-borne astronomical soft gamma-ray polarimeter optimized for point-like sources ${ }^{3)}$. It measures polarization in the energy range $25-80 \mathrm{keV}$ from sources with flux levels as low as $200 \mathrm{mCrab}$ by using the azimuthal angle anisotropy of Compton-scattered polarized photons. As shown in Figure 1 and 2, it measures coincident Compton scattering and photoabsorption for gamma-ray events in an array of 217 well-type phoswich detector cells (PDCs), each comprising a fast plastic scintillator (detection part), a slow plastic scintillator collimator and a BGO crystal (shield), which are viewed by a single photo-multiplier tube (PMT). The 217

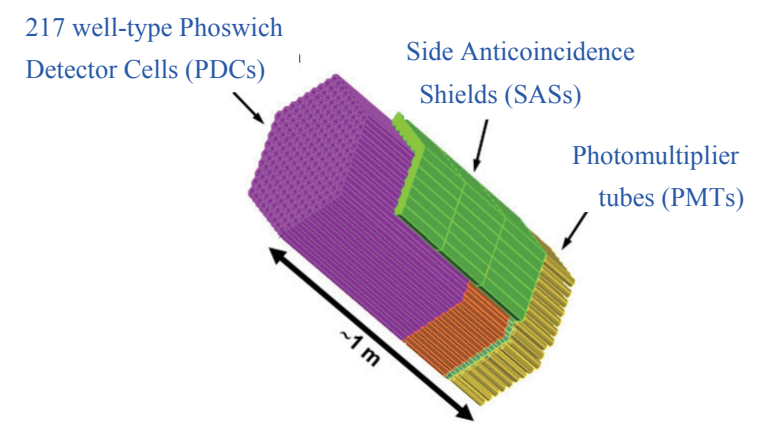

Fig. 1. Schematic view of the PoGOLite detector array, consisting of 217 PDCs and 54 SAS units, giving 271 PMT output signals in total. The field view is narrow of $1.25 \mathrm{msr}$ (FWHM) and the effective area between $25-60 \mathrm{keV}$ is large as $\sim 228 \mathrm{~cm}^{2}$ for the polarization measurements at 40 $\mathrm{keV}$. The background rate is reduced to $\sim 100 \mathrm{mCrab}$ for $25-50 \mathrm{keV}$. A lateral polyethylene shield (not shown here) is used to reduce the background from atmospheric and structure-induced neutrons. The assembly is estimated to weigh about $800 \mathrm{~kg}$. 


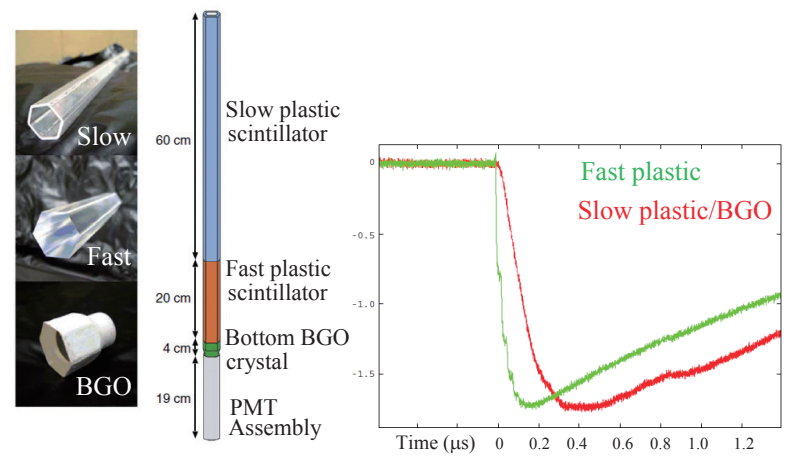

Fig. 2. (Left) Structure of the PDC. Fast, slow and BGO scintillators are viewed by one PMT. (Right) An example of PDC waveforms output from the charge-sensitive amplifier. The decay time of the fast scintillator $(\tau \sim 2$ $\mathrm{ns})$ is distinguishable from that of the slow scintillator/BGO ( $\tau \sim 300 \mathrm{~ns})$.

PDCs are surrounded by a side anti-coincidence shield (SAS) made of 54 segments of BGO crystals. An engineering "pathfinder" flight with 61-PDC and 30-SAS units is planned for 2010 from the Esrange facility in the North of Sweden.

The in-flight background due to cosmic-rays, charged particles, atmospheric gamma-rays and neutrons is extremely high, typically a few hundred $\mathrm{Hz}$ in each unit. The data acquisition (DAQ) system of PoGOLite is required to handle weak signals from astrophysical objects (200mCrab, 2-4 counts per second in the energy range of the instrument) under such a severe environment.

In the present study, we have developed the flight DAQ system with SpaceWire interface for the pathfinder flight and verified the performance through beam tests simulating the in-flight background and the polarized emission from celestial objects.

\section{Data Acquisition System of PoGOLite}

\subsection{Gamma-ray measurement by PDC units}

The PoGOLite DAQ system consists of six parts: front-end electronics, waveform digitizer, trigger logic, global event logic, microprocessor and storage system. These parts are undertaken by four electronic components: waveform digitizer board, digital I/O board, SpaceCube ${ }^{4)}$ and router board. The first three functions are implemented on the waveform digitizer board, the fourth logic on the digital I/O board, the last two in the SpaceCube, and all the electronics are inter-connected through the router board. Figures 3 and 4 show pictures of the SpaceWire boards and a block diagram of the DAQ system, respectively.

Signals from the last dynodes of all PDC and SAS PMTs are fed to individual flash ADCs (FADC) and digitized to 12-bit accuracy at a $37.5 \mathrm{MHz}$ sampling rate in waveform digitizer boards, where one digitizer board can associate up to 8 PDCs. FPGAs on the digitizer boards continuously monitor the waveforms and issue a Level-0 (L0) trigger, when a transient signal compatible with a clean hit in the fast plastic scintillator is detected above the lower discrimination level $\sim 15 \mathrm{keV}$. A veto signal is issued when the FPGA detects a transient signal above the upper discrimination level (UD veto) or one compatible with a slow rise-time corresponding to that expected from the slow plastic scintillator or BGO (Pulse-Shape Discrimination veto, PSD veto; see Figure 2).

The global event logic on-board the digital I/O board collects all L0 triggers and vetoes, and issues an acquisition request (L1 trigger) if there is no UD nor PSD veto from any PDC. When the waveform digitizer receives an L1 signal from the global event logic, it moves 15 pre-trigger and 35 post-trigger samples from each FADC to a FIFO after zero-suppression (i.e., channels of which the signals are below the threshold do not store any waveforms). The pre-trigger samples are used to correct for possible baseline shift due to preceding signals. Signals from SAS BGO modules are also stored when an L1 trigger is received. The hit pattern of the SAS signals is utilized for background rejection. The only difference between the PDC and SAS digitizer boards is the programming of the FPGA. In the final step, the microprocessor in the SpaceCube records all waveforms from both PDC and SAS digitizer boards to the storage system. Figure 5 shows waveforms for a Compton scattering and a photoabsorption, caused by a gamma-ray.

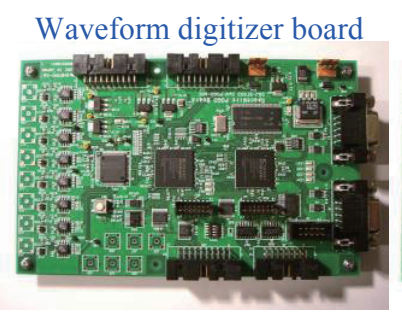

Digital I/O board
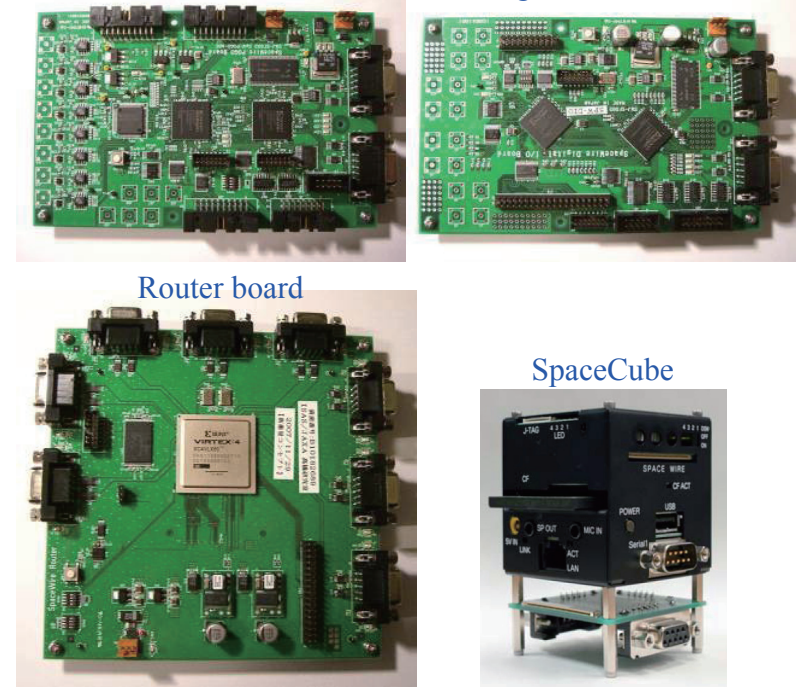

Fig. 3. SpaceWire boards and the SpaceCube developed by JAXA, NEC and Shimafuji Electric. (Top left) waveform digitizer board, (Top right) digital I/O board, (Bottom left) router board. All the components are operated with $+5 \mathrm{~V}$ input.

For the 217-PDC PoGOLite, the L0 trigger, the UD rate and the PSD veto rates are estimated to be about $13 \mathrm{kHz}, 6 \mathrm{kHz}$ and $12 \mathrm{kHz}$, respectively. We expect the $\mathrm{L} 1$ rate to be $\sim 0.5$ $\mathrm{kHz}$, with a dead time fraction of $\sim 0.6 \%$. The validity of the UD and PSD vetoes were confirmed with a proton beam at the Research Center for Nuclear Physics (RCNP) in Osaka University, with the proton rates of up to $\sim 5 \mathrm{kHz}$, which is more than an order of magnitude higher than that expected for one PDC unit in flight.

The data size of each waveform is 110 bytes, which includes a 10-byte header as well as the 50-clock waveform (15 pre-samples and 35 post-samples). Each one-clock 


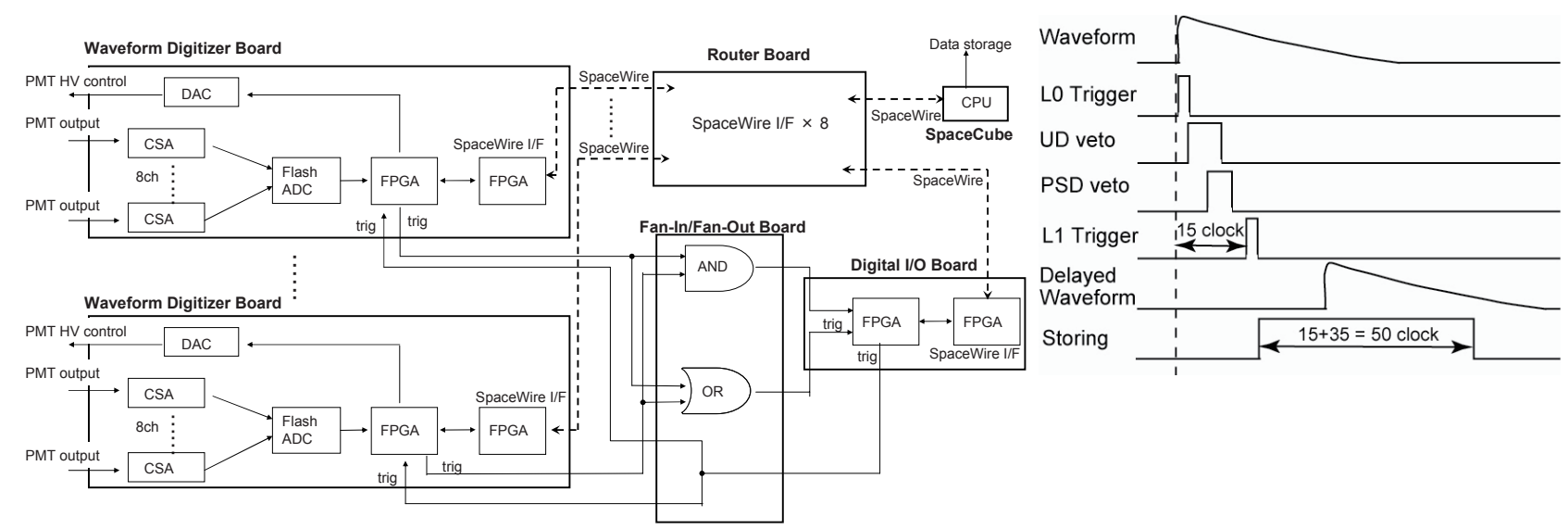

Fig. 4. (Left) Block diagram of the PoGOLite DAQ system. All waveform digitizer boards and digital I/O boards are controlled and read by the SpaceCube through the router board. The reference voltage to operate each PMT (around $+5 \mathrm{~V}$ ) is set by accessing the waveform digitizer board through the SpaceWire connection. (Right) Timing chart of the DAQ system. L0 trigger without UD/PSD vetoes from 217 PDC units (waveform digitizer boards) issues L1 trigger (digital I/O board) to start storage of waveforms.

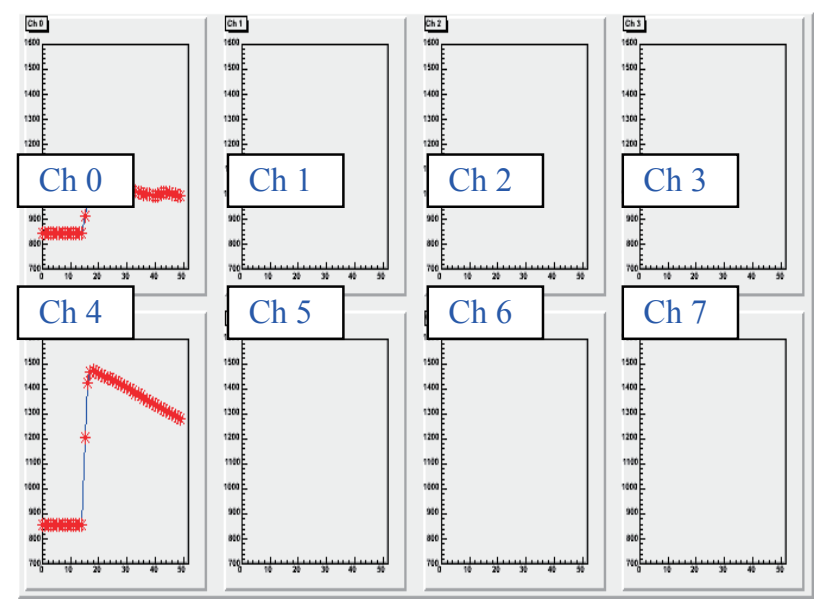

Fig. 5. An example of waveforms from a gamma-ray. One photon has Compton-scattered in channel 0 (top left) and subsequently been photo-absorbed in channel 4 (bottom left) of the same waveform digitizer board. Signals from the remaining six channels were not stored due to the zero-suppression setting.

waveform consists of a 12-bit pulse height and a 4-bit dummy column for a total of 16 bits ( 2 bytes). The header includes a sequential number, the logical address of the waveform digitizer board, an 8-channel hit-pattern for the board, presences of UD and PSD vetoes, and a 32-bit time counter (one count is $0.427 \mu \mathrm{s}$ ). During data acquisition, measured waveforms are first stored in the FPGA of each digitizer board. They are then read out by the SpaceCube through the SpaceWire connection when more than 32 waveforms are stored in one board. This reduces the required number of the read-out accesses by the SpaceCube and increases the data-acquisition rate. With this configuration, we have at present obtained a maximum data-acquisition rate of about 400 waveforms per second, and this rate is sufficient for the

\section{1-unit pathfinder flight planned in 2010.}

To support waveform sampling technique described above, the digital I/O board additionally outputs a pseudo-trigger every second if it is not occupied with data acquisition. Once the pseudo-trigger is issued, every waveform digitizer board receives this signal and simultaneously stores a pseudo event, from which we can synchronize the on-board time counters among the SpaceWire boards and estimate the dead time (from the number of the discarded events) in off-line analyses.

\subsection{Background monitoring by SAS units}

As well as storing hit-pattern information for background events coincident with the L1 trigger (see section 2.1), the waveform digitizer board for the SAS continuously records a Pulse Height Analysis (PHA) histogram with a 12-bit resolution, where the exposure of the histogram is changeable. This histogram can be used to study the in-flight background environment, which changes from moment to moment. To obtain an accurate energy spectrum from the PHA, we have implemented a base-line subtraction logic in the FPGA of the SAS digitizer board ${ }^{5)}$.

As shown in Figure 6 top, the FPGA subtracts a "delayed" waveform from the original one and issues a trigger when the differential pulse-height exceeds a given threshold. It then subtracts the base-line (pre-trigger pulse height) and records the "corrected" pulse height in a PHA histogram. This logic allows us to measure the pulse-height (photon energy) correctly, even if a signal occurs during the undershoot of a large pulse caused e.g. by charged particles. To verify the performance of this logic, we obtained spectra of low-intensity gamma-rays from ${ }^{241} \mathrm{Am}(59.5 \mathrm{keV})$ in a high-intensity background from $662 \mathrm{keV}$ gamma-rays from ${ }^{137} \mathrm{Cs}$, which simulates gamma-ray background. The result shown in Figure 6 bottom confirms the validity of the logic and demonstrates that the SAS unit can reject gamma-rays down to $\sim 50 \mathrm{keV}$.

We also studied changes in the SAS PHA histogram caused by large signals from charged particles at RCNP. In these tests, SAS PHA spectra of $662 \mathrm{keV}$ gamma-rays from ${ }^{137} \mathrm{Cs}$ were obtained in a background from $392 \mathrm{MeV}$ protons. The spectra of ${ }^{137} \mathrm{Cs}$ are shown in Figure 7, where the $662 \mathrm{keV}$ peak was unaffected even with a proton intensity of up to $6.5 \mathrm{kHz}$. This rate is significantly higher than that expected for one SAS unit in flight $(\sim 1 \mathrm{kHz})$, confirming the performance of the SAS PHA at float altitude $(\sim 40 \mathrm{~km})$. 

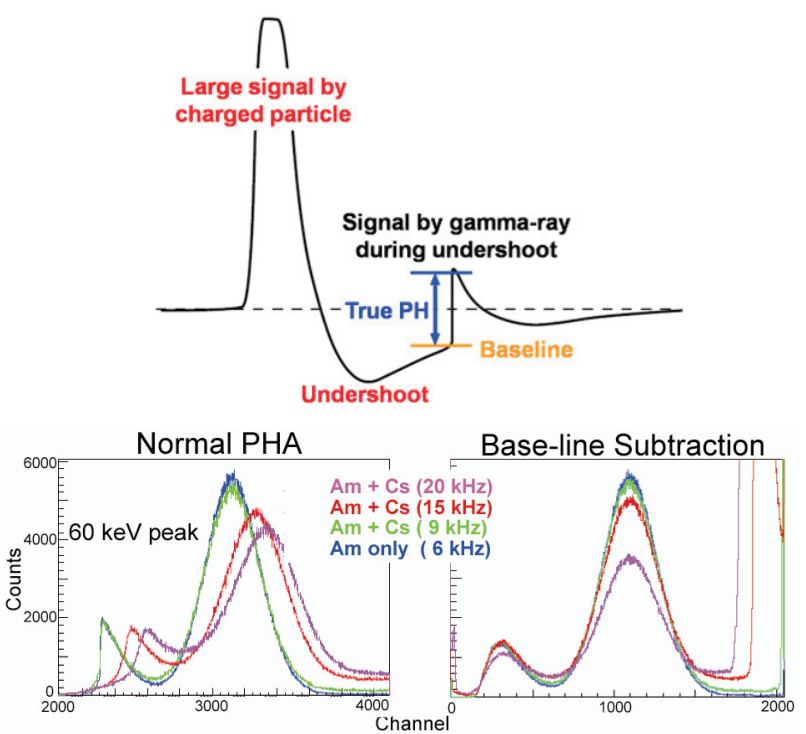

Fig. 6. (Top) Base-line subtraction in the SAS PHA monitoring. (Bottom) Spectra of low-intensity gamma-rays from ${ }^{241} \mathrm{Am}(59.5 \mathrm{keV})$, recorded under irradiation of high-intensity gamma-rays from ${ }^{137} \mathrm{Cs}(662$ $\mathrm{keV}$ ). The peak in the left spectrum (without base-line subtraction) is shifted due to the gamma-rays from ${ }^{137} \mathrm{Cs}$, but in the right spectrum, the peak position remains constant thanks to the base-line subtraction.

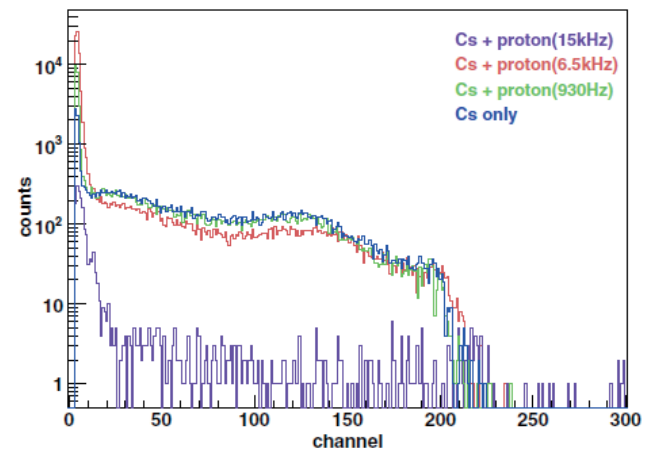

Fig. 7. SAS PHA spectra of ${ }^{137} \mathrm{Cs}(662 \mathrm{keV})$ measured under irradiation with $392 \mathrm{MeV}$ proton beams with various intensities.

\section{Results of KEK Beam Test with 19 PDCs and One SAS Unit}

The performance of the detector units and the flight-version DAQ system was verified in a beam test, carried out at KEK-PF in $2008^{6}$. In this test, a prototype instrument, comprising 19 PDCs and one SAS unit, was irradiated by a pencil beam of $50 \mathrm{keV}$ X-ray (polarization degree of $\sim 90 \%$ ). As shown in Figure 8, signals from all 20 units were fed to 4 waveform digitizer boards (three for the 19 PDCs and one for the SAS). The DAQ was constructed as described in section 2.1 with a digital I/O board, a SpaceCube and a router board. This setup is identical to those of the 61-PDC pathfinder instrument and the full 217-PDC one, apart from the number of the waveform digitizer boards. Therefore, once the procedure is established with this 19-PDC assembly, it is straight-forward to expand the DAQ system.
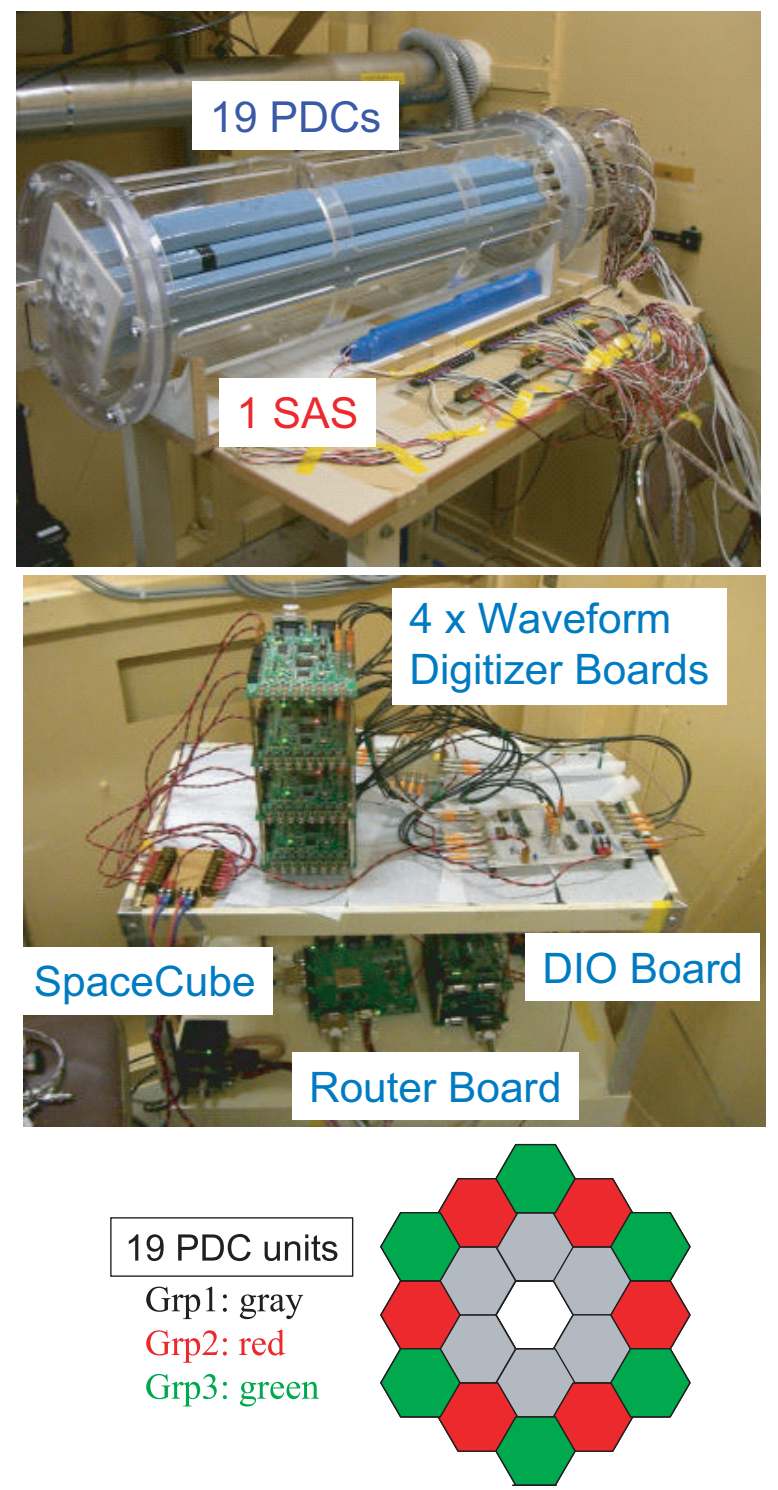

Fig. 8. (Top) Prototype instrument consisting of 19 PDCs and one SAS unit, used in the KEK beam test. (Middle) DAQ Setup with the flight-version SpaceWire boards. PMT voltages are also controlled by the digitizer boards. (Bottom) Arrangement of the 19 PDCs. A polarized beam is irradiated at the center unit.

Candidates of X-ray events with both a Compton scattering and a photoabsorption were selected from the recorded PDC waveforms in off-line analyses. The pulse-height thresholds were achieved as 0.3 photo-electron $(\sim 0.5 \mathrm{keV})$ and $15 \mathrm{keV}$ for the Compton and photoabsorption events, respectively. Between successive measurements, the instrument was rotated in 30-degree steps until a full revolution had been completed. The flight-version of the instrument will be rotated in a similar fashion in order to remove systematic bias. We then determined the azimuthal angle of scattering for each event, corrected it for the rotation of the detector, and derived the modulation curve. Figure 9 shows the measured modulation curve, where distributions of valid Compton events along the azimuth angle are drawn separately for three PDC groups, Grp 1, Grp 2 and Grp3 (see Figure 8 bottom). The modulation 
depends on the distance between the Compton-scattering site and the photoabsorption site. Obtained modulation factors for the $90 \%$ polarized beam are $(31.3 \pm 0.4) \%,(37.9 \pm 0.7) \%$ and (40.2 \pm 0.8$) \%$ for Grp1, 2 and 3, respectively, and (34.1 \pm $0.3) \%$ when combined over the three groups. These results are consistent within $\sim 5 \%$ with those predicted by our GEANT4-based simulation, which is also utilized for the development of the detector response for observations during the balloon flight.

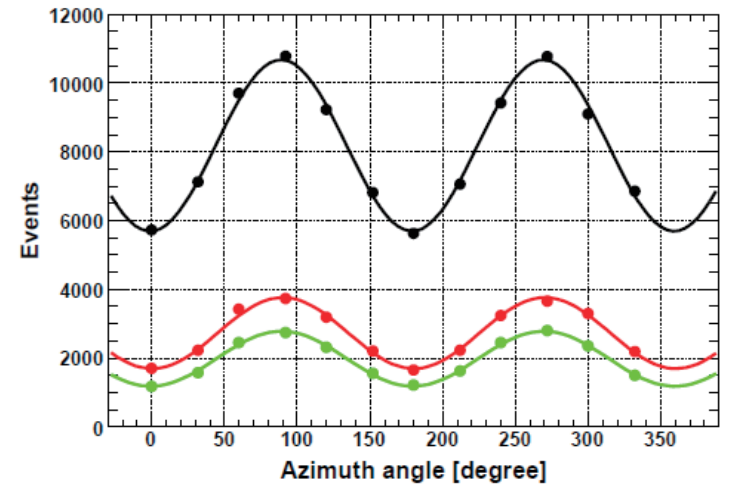

Fig. 9. Measured modulations of a $\sim 90 \%$ polarized beam with an energy of $50 \mathrm{keV}$. The curves are obtained from the three PDC sets, Grp1 (black), Grp2 (red), and Grp3 (green), shown in the bottom of Figure 8.

\section{Conclusions}

The flight-version DAQ system of PoGOLite has been developed based on the SpaceWire interface with consisting of the waveform digitizer board, digital I/O board, router board and microprocessor. The performance was tested through the beam test at KEK-PF, where a $50 \mathrm{keV}$ X-ray beam with a $90 \%$ polarization degree was irradiated. During the test, this system worked well, and the modulation factor of the detector was measured as $\sim 34 \%$, which is consistent with the simulation.

From these results, we have evaluated the performance of the DAQ system to operate the 61-PDC and 30-SAS units onboard the pathfinder flight launched in 2010 from the Esrange facility, and confirmed the capability to detect the polarization of the soft gamma-ray from celestial objects.

\section{Acknowledgments}

The SpaceWire-based I/O and boards were developed in JAXA's program "Research and Development for Future Innovative Satellite.”

\section{References}

1) Weisskopf, M. C. et al.: A Precision Measurement of the X-ray Polarization of the Crab Nebula without Pulsar Contamination, Astrophysical Journal, 220 (1978), pp.L117-L121.

2) Dean, A. J. et al.: Polarized Gamma-ray Emission from the Crab, Science, 321 (2008), pp.1183-1185.

3) Kamae, T. et al.: PoGOLite - A high sensitivity balloon-borne soft gamma-ray polarimeter, Astroparticle Physics, 30 (2008), pp. 72-84.

4) Takahashi, T. et al.: Space Cube 2 - an Onboard Computer based on Space Cube Architecture, Proceedings of 1st international SpaceWire conference, 2007.

5) Tanaka, T. et al.: Data acquisition system for the PoGOLite astronomical hard X-ray polarimeter, IEEE NSS conference record (2007), pp. 445-449.

6) Takahashi, H. et al.: Beam test results of the polarized gamma-ray observer, PoGOLite, IEEE NSS conference record (2008), pp. 732-736. 